\title{
La Evaluación En El Proceso De Enseñanza- Aprendizaje En La Asignatura De Inglés
}

\author{
Rodríguez Arellano Nancy Georgina
}

Magister en docencia universitaria y administración de la educación Docente del Centro de idiomas de la Escuela Superior Politécnica de

Chimborazo, Ecuador

\section{Enrique Jesús Guambo Yerovi}

Magister en Lingüística Aplicada al Aprendizaje del Idioma Inglés.

Docente de la Escuela Superior Politécnica de Chimborazo,

Centro de Idiomas, Ecuador

Doi: 10.19044/esj.2018.v14n11p47 URL:http://dx.doi.org/10.19044/esj.2018.v14n11p47

\begin{abstract}
The objective of the present research is to determine the incidence of the evaluation in the academic performance of the students of the ninth years of Basic Education in the subject of English. For this, the strengths and weaknesses of the evaluation were identified as part of the pedagogical, didactic strategies applied, the teacher's performance in the classroom, the interpersonal relationships and the treatment or approach that the teachers of the specialty demonstrate with the students. The research was based on the critical-propositive paradigm. A review of the literature on pedagogical models and its impact on the evaluation of learning was made, to then establish the level of relationship between teacher and student in the teaching-learning process. The modality, type and level were identified with a global vision of cognitive totality and/or of skills, objectives, hypotheses and operationalization of variables. Through a questionnaire, the information was collected and a logical, mathematical and statistical model was established in order to demonstrate the impact of evaluation on learning, taking as a reference the desired and expected frequencies. It was established that Teachers by means of active techniques developed and impacted through the evaluation on meaningful knowledge.
\end{abstract}

Keywords: Incidence, Evaluation, Teaching-learning process, English

\section{Resumen}

La presente investigación tiene como objetivo determinar la incidencia de la evaluación en el rendimiento académico de las y los estudiantes de los 
novenos años de Educación Básica en la asignatura de inglés. Para ello, se identificó las fortalezas y debilidades de la evaluación como parte de lo pedagógico, estrategias didáctica aplicadas, actuación del docente en el aula, las relaciones interpersonales y el trato o acercamiento que los docentes de la especialidad demuestran con los estudiantes. La investigación se basó en el paradigma crítico-propositivo. Se realizó la revisión de la literatura sobre los modelos pedagógicos y su incidencia en la evaluación de los aprendizajes, para luego establecer el nivel de relación entre docente y estudiante en el proceso enseñanza-aprendizaje. Se identificó la modalidad, tipo y nivel con una visión global de totalidad cognitiva y/o de destrezas, objetivos, hipótesis y operacionalización de variables. A través de un cuestionario se recolectó la información y se estableció un modelo lógico, matemático y estadístico a fin de demostrar la incidencia de la evaluación en el aprendizaje, tomando como referencia las frecuencias deseadas y esperadas. Se estableció los docentes a través de técnicas activas desarrollaron e incidieron a través de la evaluación en el conocimiento significativo.

Palabras claves: Evaluación, Proceso de enseñanza- aprendizaje, Inglés

\section{Introducción}

En los últimos treinta años, se ha modificado los criterios que se tenían acerca de la evaluación, quedando atrás el caduco concepto del cual se tomaba únicamente el nivel de conocimientos; resultado que dependía la promoción o pérdida del año, es decir era vertical.

En la actualidad, se cuestiona un tema de gran importancia para nuestra realidad y desarrollo de nuestro país tanto en el campo científico como en el tecnológico, este cuestionamiento se refiere al área educativa, en el aspecto de la evaluación de los aprendizajes.

En los momentos actuales la evaluación es holística e integral, se toma en cuenta el desempeño del profesor, los recursos, metodología, habilidades destrezas de los estudiantes, valores a lo que se lo conoce como competencias.

La evaluación se halla en la "encrucijada" didáctica, pues se considerar como efecto y causa del aprendizaje. Al respecto Miller sostiene que la evaluación orienta el currículum y puede, generar un cambio en los procesos de aprendizaje. Barberá (1999), Allen (2000), McDonald (2000) Dochy (2002) o Bain (2006) han ayudado a entender, que la evaluación no puede limitarse solo a la calificación (sino que ésta es un subconjunto de la evaluación); no puede centrarse en el recuerdo y la repetición de información (sino que se deben de evaluar habilidades cognitivas de orden superior) y que no puede limitarse a una evaluación tradicional con "lápiz y papel", sino que se requieren instrumentos complejos y variados ayudados de tecnologìa. 
La evaluación debe constituir una oportunidad de aprendizaje y no utilizarse para seleccionar ciertas competencias, sino para promoverlas en todos los estudiantes. Esta dimensión formativa formulada por Scriven ha sido abordada ampliamente en los últimos años por Hall y Burke (2003) y Kaftan (2006).

La evaluación debe concientizar a los estudiantes para determinar cuál es su nivel de competencias, de cómo resuelven las tareas y de qué puntos fuertes deben potenciar y qué puntos débiles deben corregir para enfrentarse a situaciones de aprendizaje futuras. Este proceso de autorregulación, tratado, entre otros, por Boekaerts, Pintrich y Zeidner (2000) va a ser esencial para seguir aprendiendo a lo largo de toda la vida (Life Long Learning) y, constituye en sí mismo, una competencia clave.

La evaluación ha de ser coherente con el resto de elementos del diseño formativo, ha de hallarse integrada en el mismo. Por ello las experiencias metodológicas más coherentes con los diseños por competencias, como son las simulaciones, los proyectos, el PBL,... llevan asociadas actividades evaluativas muy relevantes para la evaluación por competencias. Los trabajos de Segers y Dochy (2001) o de Gijbels et al. (2005) han resultado muy ilustrativos en este punto.

"En los primeros años del siglo XX, las escuelas eran concebidas como fábricas; los estudiantes como materia prima; mientras que los conceptos educativos de conocimientos, valores y relaciones sociales se reducían a términos de neutralidad técnica y a un razonamiento estricto de medios - fines. Cuando la teoría tuvo que traducirse en metodología, las cuestiones sobre valores morales y éticos se enmarcaron dentro de una lógica del conocimiento "científico" y de la organización burocrática" (Giroux 2003).

La presente investigación se fundamenta en investigaciones como: "La evaluación como acreditación de los aprendizajes en el Colegio Particular de Educación a Distancia "República de Argentina" Lcdo. Javier Patiño.- Lcda. Blanca Morocho." El par didáctico evaluación-acreditación implica un proceso continuo de valoración en lo conceptual, procedimental y actitudinal de los estudiantes, a fin de certificar el desarrollo efectivo de habilidad y destrezas que le permitan estructurar su propio proyecto de vida".

Así también, en la investigación denominada "Incidencia de la aplicación de la evaluación al docente en el nivel de rendimiento de los estudiantes de la especialización de contabilidad de costos y administración del Instituto Superior 24 de Mayo del Distrito Metropolitano de Quito en el año lectivo 2001 - 2002." La investigación se orientó a una evaluación de desempeño docente como un proceso de crecimiento personal y profesional, más no de forma coercitiva, la misma que determinó que incide positivamente en los aprendizajes significativos de los estudiantes" 
La educación es el resultado de mecanismos y actividades que deben generar conocimiento, desarrollo de habilidades y destrezas, así como el de cultivar valores en el proceso de conocer la realidad y contribuir a su transformación. En la educación actúa la familia, como el núcleo social en donde el/a niño/a o el/a joven son educados para convivir en comunidad como entes positivos para sí mismo y los que los rodean; por su parte el maestro, debe convertirse en un facilitador, mediador, estimulador de experiencias vitales, contribuyendo de esta manera al desarrollo de capacidades al pensar, reflexionar sentir, actuar, y promoviendo el aprendizaje por descubrimiento y significación, conduciendo al desarrollo de habilidades cognitivas; y el estudiante siendo un sujeto activo, participativo, creador, investigador, constructor, reconstructor, para que él mismo elabore progresiva, secuencial y significativamente los aprendizajes.

Durante el proceso educativo constructivista, participan elementos bio-sico-socio-históricos-culturales, el verdadero aprendizaje se realiza cuando el individuo es capaz de incorporar los nuevos conocimientos para mejorar su entorno, cambiar la calidad de vida y producir cultura.

E1 aprendizaje es una actividad social dirigida a cambiar la naturaleza, la sociedad, y la personalidad humana que requiere de estrategias cognitivas de exploración, adquisición y reflexión; así como de planificación y regulación para que el estudiante aprenda. Las estrategias ha emplear deben respondan con las características personales de cada persona, que son la base del desarrollo de las habilidades y destrezas intelectuales. Por lo mismo, no es posible percibir un modo de enseñanza único para favorecer el aprendizaje significativo. Es responsabilidad de las instituciones educativas formar individuos pensantes, críticos, reflexivos, solidarios, solucionadores de problemas a través de un proceso de aprendizaje en el que predomine permanentemente la coparticipación diálogo y respeto como persona.

De lo mencionado anteriormente los docentes que orientan la materia de inglés en los novenos años y otros paralelos, deben desarrollar sus capacidades y habilidades para contribuir a la consolidación del proceso formativo, eliminando los verbos medir o controlar, apareciendo verbos pedagógicos de acompañamiento como estimular, favorecer, identificar, para así contribuir en el crecimiento en todas las dimensiones del estudiante.

En la presente investigación se denota la topología de la evaluación, contemplando su orientación, regulación y certificación, orientándola de un punto de vista diagnóstica, preventiva y predictiva.

En la evaluación diagnóstica se regularon las tareas en marcha o en proceso; mientras que en la evaluación preventiva, se aplicó a la realidad y se buscó información para predecir hechos futuros, permitiendo elaborar hipótesis sobre acontecimientos; mientras que predictivamente se anticipó 
cómo será el desempeño de un individuo; es decir, se pronosticó que posibilidad de éxito promueve sus acciones futuras de compensación.

Con respecto a la evaluación de regulación, se ajustó el proceso de enseñanza sobre la marcha, buscando información sobre procedimientos, que en caso de ser necesario fueron corregidos a través de estrategias de los docentes, como material didáctico y actividades de interacción.

En la evaluación de certificación, se analizó los resultados del aprendizaje. Para ello, la selección implicó la clasificación de los sujetos en función del rendimiento; mientras que la evaluación de tipo acumulativa, permitió conocer el balance final centrándose en los logros alcanzados.

\section{Metodología}

La investigación tiene un enfoque que se orienta en un paradigma de actualidad, que busca la comprensión de fenómenos sociales, asume una realidad dinámica y estable en el tiempo. La investigación es de campo, ya que estudió los hechos en el lugar donde se suscitan los mismos.

La investigación es descriptiva, ya que se cotejaron dos fenómenos representadas a través de la variable dependiente e independiente. También es explicativa, pues se realizó la comprobación de la hipótesis.

La investigación se orientó con un paradigma Crítico - Propositivo, con visión de totalidad concreta, ya que se realizó una explicación y para determinar una solución al problema concreto, en este caso la evaluación de los aprendizajes. La evaluación se fundamentó en el mecanicismo, realismo, un cierto determinismo y el objetivismo.

La investigación se realizó en la Unidad Educativa "Julio María Matovelle" de la ciudad de Quito, con los estudiantes y docentes de los novenos años de Educación Básica en la asignatura de inglés; mientras que la muestra representa 97 estudiantes y 2 docentes entre hombres y mujeres.

Para lograr la meta, se elaboró una guía de evaluación de los aprendizajes basadas en el Modelo Pedagógico Constructivista aplicado a la materia de inglés para el aprendizaje activo de los estudiantes; mientras que para el uso adecuado de la guía, se realizó una capacitación dirigida a los docentes, a fin de que se transmitan los conocimientos y se ponga práctica las técnicas activas en el desarrollo del conocimiento significativo.

Como técnica se utilizó la entrevista a través de un cuestionario, que fue el instrumento para la obtención de la información, aplicándola a docentes y estudiantes. El mismo que fue sometido a una prueba piloto para determinar su validez y confiabilidad; que finalmente fue aplicado en un lapso de dos días.

Los datos recogidos fueron sometidos a verificación para comprobar que han sido adecuadamente contestados. En el caso de cuestionarios incompletos o incorrectos se procuró nuevamente aplicarlos y en caso de persistir se eliminaba completamente. 
Verificados los cuestionarios se procedió a la tabulación de la información. Se realizó el reajuste en la tabulación en caso de dimensiones o casillas vacías o con datos sin peso significativo. Posterior se convirtieron los datos obtenidos en porcentajes alcanzados y se procedió con la representación gráfica para continuar con el análisis estadístico y su respectiva interpretación.

A través de un modelo lógico se estableció una hipótesis, y se optó por un modelo matemático para su análisis a través del CHI cuadrado y gracias a la estadística se decidió y verificó la hipótesis planteada en la presente investigación de los datos de frecuencias esperadas y observadas.

\section{Resultados:}

Tabla 1. Resultados aplicados a los estudiantes del Noveno A y B

\begin{tabular}{|c|c|c|c|c|c|c|c|c|c|c|c|c|c|c|c|c|}
\hline \multirow[b]{3}{*}{ Escala de apreciación } & \multicolumn{8}{|c|}{ Noveno A } & \multicolumn{8}{|c|}{ Noveno B } \\
\hline & \multicolumn{2}{|c|}{ 离 } & \multicolumn{2}{|c|}{ 预 } & \multicolumn{2}{|r|}{ 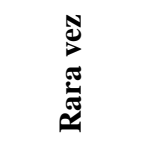 } & \multicolumn{2}{|r|}{$\begin{array}{l}\stackrel{\Xi}{\Xi} \\
\text { Z }\end{array}$} & \multicolumn{2}{|c|}{ 离 } & \multicolumn{2}{|c|}{ 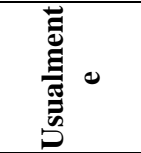 } & \multicolumn{2}{|r|}{ 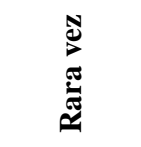 } & \multicolumn{2}{|r|}{ 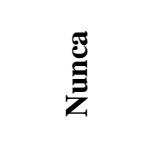 } \\
\hline & $\mathbf{N}$ & $\%$ & $\mathbf{N}$ & $\%$ & $\mathbf{N}$ & $\%$ & $\mathbf{N}$ & $\%$ & $\mathbf{N}$ & $\%$ & $\mathbf{N}$ & $\%$ & $\mathbf{N}$ & $\%$ & $\mathbf{N}$ & $\%$ \\
\hline Pregunta 1 & 5 & 11,90 & 15 & 35,71 & 17 & 40,48 & 5 & 11,90 & 0 & 0,00 & 2 & 4,17 & 17 & 35,42 & 29 & 60,42 \\
\hline Pregunta 2 & 2 & 4,76 & 6 & 14,29 & 15 & 35,71 & 19 & 45,24 & 0 & 0,00 & 12 & 25,00 & 36 & 75,00 & 0 & 0,00 \\
\hline Pregunta 3 & 33 & 78,57 & 8 & 19,05 & 1 & 2,38 & 0 & 0,00 & 41 & 85,42 & 7 & 14,58 & 0 & 0,00 & 0 & 0,00 \\
\hline Pregunta 4 & 10 & 23,81 & 4 & 9,52 & 10 & 23,81 & 18 & 42,86 & 0 & 0,00 & 3 & 6,25 & 4 & 8,33 & 41 & 85,42 \\
\hline Pregunta 5 & 6 & 14,29 & 14 & 33,33 & 15 & 35,71 & 7 & 16,67 & 9 & 18,75 & 24 & 50,00 & 13 & 27,08 & 2 & 4,17 \\
\hline Pregunta 6 & 2 & 4,76 & 6 & 14,29 & 21 & 50,00 & 13 & 30,95 & 0 & 0,00 & 0 & 0,00 & 14 & 29,17 & 34 & 70,83 \\
\hline Pregunta 7 & 0 & 0,00 & 0 & 0,00 & 3 & 7,14 & 39 & 92,86 & 0 & 0,00 & 8 & 16,67 & 39 & 81,25 & 1 & 2,08 \\
\hline Pregunta 8 & 5 & 11,90 & 7 & 16,67 & 12 & 28,57 & 18 & 42,86 & 3 & 6,25 & 30 & 62,50 & 9 & 18,75 & 6 & 12,50 \\
\hline Pregunta 9 & 0 & 0,00 & 1 & 2,38 & 2 & 4,76 & 39 & 92,86 & 0 & 0,00 & 0 & 0,00 & 0 & 0,00 & 48 & 100,00 \\
\hline Pregunta 10 & 12 & 28,57 & 24 & 57,14 & 6 & 14,29 & 0 & 0,00 & 0 & 0,00 & 2 & 4,17 & 35 & 72,92 & 11 & 22,92 \\
\hline
\end{tabular}

Fuente: Cuestionario

Elaborado por: Rodríguez Nancy

\section{Planteamiento de la Hipótesis}

\section{Modelo Lógico}

Ho: La Evaluación no incide significativamente en el proceso de enseñanza aprendizaje en los novenos años de Educación Básica en la asignatura de Ingles de la Unidad Educativa "Julio María Matovelle" de la Ciudad de Quito, durante el año lectivo 2005-2006

Ha: La Evaluación si incide significativamente en el proceso de enseñanza aprendizaje en los novenos años de Educación Básica en la asignatura de Ingles de la Unidad Educativa "'Julio María Matovelle" de la ciudad de Quito, Durante El Año Lectivo 2005-2006.

Modelo Matemático

Ho: $\mathrm{O}=\mathrm{E}$

Ha: $\mathrm{O} \neq \mathrm{E}$
Modelo Estadístico

$$
x^{2}=\sum\left[\frac{(o-E)^{2}}{E}\right]
$$


Nivel de significación
$\alpha=0,05$
95\% de confiabilidad
$5 \%$ de error

\section{Zona de rechazo de la $\mathrm{Ho}$}

$\mathrm{G} 1=(\mathrm{C}-1)(\mathrm{F}-1)=(4-1)(2-1)=3$

Para G1 $=3 x^{2}=7.815$ (tomado desde la tabla)

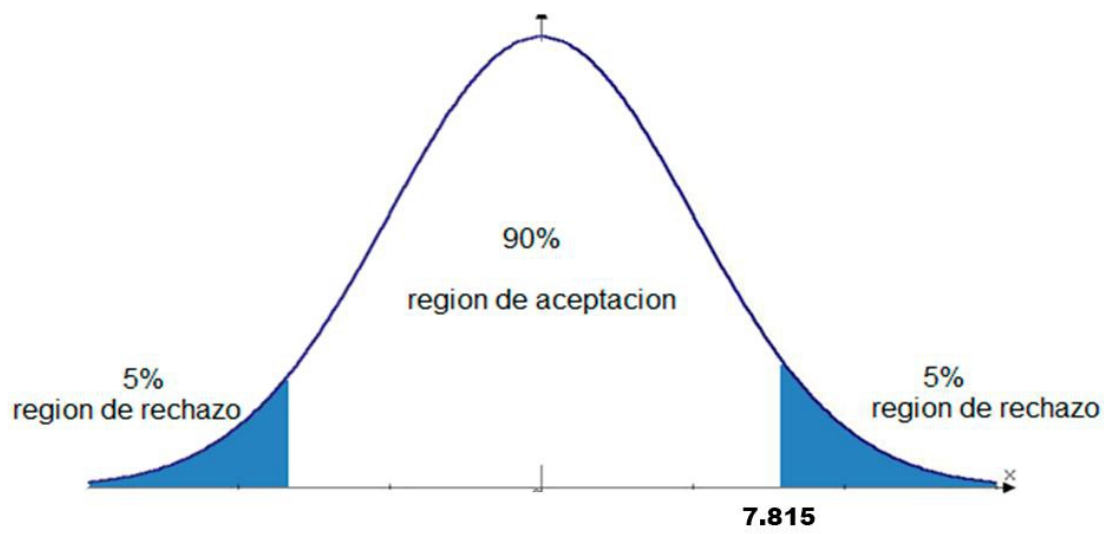

Gráfico 1: Calculo estadístico

\section{Regla de decisión}

Se rechaza la H0 si chi cuadrado calculado es mayor de 7,815 correspondiente al valor de la tabla

$\mathrm{R}(\mathrm{Ho}) x^{2}>7.815$

\section{Cálculo estadístico}

Datos obtenidos de la investigación: 10 preguntas realizadas a los estudiantes del 9A y 9B sobre la incidencia de la evaluación en el proceso de enseñanza aprendizaje del idioma inglés.

Tabla 1. Frecuencias Observadas

\begin{tabular}{|c|c|c|c|c|c|}
\hline Escala de operación & Siempre & Usualmente & Rara vez & Nunca & TOTAL \\
\hline Evaluación & 86 & 40 & 66 & 78 & 270 \\
\hline $\begin{array}{c}\text { Proceso enseñanza } \\
\text { aprendizaje }\end{array}$ & 42 & 133 & 203 & 252 & 630 \\
\hline TOTAL & 128 & 173 & 269 & 330 & 900 \\
\hline
\end{tabular}

Fuente: Encuesta a estudiantes

Elaborado por: Rodríguez Nancy

Tabla 2. Frecuencias Esperadas

\begin{tabular}{|c|c|c|c|c|c|}
\hline Escala de operación & Siempre & Usualmente & Rara vez & Nunca & TOTAL \\
\hline Evaluación & 38.4 & 51.9 & 80.7 & 99 & 270 \\
\hline $\begin{array}{c}\text { Proceso enseñanza } \\
\text { aprendizaje }\end{array}$ & 89.6 & 121.1 & 188.3 & 231 & 630 \\
\hline TOTAL & 128 & 173 & 269 & 330 & 900 \\
\hline
\end{tabular}

Fuente: Encuesta a estudiantes

Elaborado por: Rodríguez Nancy 
Tabla 3. Calculo Chi - Cuadrado

\begin{tabular}{|c|c|c|}
\hline O & E & $\frac{(\boldsymbol{o}-\boldsymbol{E})^{\mathbf{2}}}{\boldsymbol{E}}$ \\
\hline 86 & 38.4 & 59,00 \\
\hline 42 & 89.6 & 25.29 \\
\hline 40 & 51.9 & 2.73 \\
\hline 133 & 121.1 & 1.17 \\
\hline 66 & 80.7 & 2.68 \\
\hline 203 & 188.3 & 1.15 \\
\hline 78 & 99 & 4.45 \\
\hline 252 & 231 & 1.99 \\
\hline
\end{tabular}

Fuente: Encuesta a estudiantes

Elaborado por: Rodríguez Nancy

\section{Decisión estadística}

Con tres grados de libertad y $95 \%$ de confiabilidad, aplicando la prueba $x^{2}$, se tiene que el valor tabular es igual 7.815; de acuerdo a los resultados obtenidos con los datos tomados de la encuesta, se ha calculado el valor de $x^{2}$ que alcanza a 98.38; lo que implica que se rechaza la hipótesis nula, aceptando la hipótesis alterna que dice: La Evaluación si incide significativamente en el proceso de enseñanza aprendizaje en los novenos años de Educación Básica en 1a asignatura de Ingles de la Unidad Educativa "Julio María Matovelle" de la ciudad de Quito, durante el Año Lectivo 2005-2006.

\section{Discusión}

E1 ámbito educacional, constituye un reto entre las metas que fijan su cometido y el nivel de resultados alcanzados. Es evidente que en el campo educativo ecuatoriano, la cultura de la evaluación es muy incipiente, convirtiéndose en un proceso de medición de aprendizajes; poniendo en práctica una corriente de poder, pues el docente tradicional maneja el sistema de evaluación de manera coercitiva, lo cual le permite superar la falta de estrategias metodológicas que apunte a un trabajo eficaz en el aula.

La evaluación consiste en llevar a cabo juicios acerca del avance y progreso de cada estudiante. Recientemente los fines de la evaluación valoran tanto el proceso de aprendizaje como los logros de los estudiantes.

En este sentido, el profesor debe mostrar una actitud positiva e imparcial hacia el proceso de evaluación del aprendizaje. La evaluación debe ser considerada como la toma de decisión del docente y su deber es aplicar la evaluación continua, que se realiza con otro tipo de medios, entre los que se incluye el conjunto de tareas realizadas por el estudiante durante el curso. Así, 1a evaluación se realiza generalmente para obtener una información más global. En el proceso de evaluación el docente debe aplicar una buena 
motivación para que el estudiante aprenda a pensar en inglés, y por ende hablar. De acuerdo a su realidad y a sus problemas.

Los docentes que no aplican la evaluación no desarrollan el espíritu científico, crítico y creativo en los estudiantes, desarrollando sujetos acríticos, pasivos, superficiales, se limita a la simple transmisión de contenidos haciendo del estudiante un objeto receptor y actuando el docente como el único conocedor y dueño de la verdad.

La tarea del docente no puede ser la misma en una concepción constructivista que en una concepción reproductora del conocimiento. La responsabilidad del docente, no es la misma en una perspectiva centrada en el estudiante y en su aprendizaje que en una perspectiva centrada en la enseñanza $\mathrm{y}$ en el docente.

Si el docente es resistente al cambio y solo actúa de acuerdo a las exigencias actuales tendrá el tipo de estudiante tradicionalista, repetitivo y no reflexivo ni activo, es decir que el estudiante continuará en estado pasivo, memorista, mecánico, individualista por lo tanto dependiente.

El docente resistente al cambio, continuará actuando de un modo impositivo, desactualizado, autoritario, verbalista, arbitrario, repetitivo, enciclopedista, dictador, transmisor de conocimientos, sin desarrollar las competencias lingüísticas en el manejo del idioma inglés. Tampoco prestará atención a los sistemas que integran el lenguaje como: sintáctico, semántica, fonológico y morfológico, situación que desprestigiará a la institución, porque el estudiante no estará en capacidad de responder a las exigencias del desarrollo nacional y mundial, estando acorde con la realidad económica, social y cultural, el idioma inglés entre otros aprendizajes significativos constituye un medio de comunicación universal para el desarrollo de la ciencia y la tecnología al servicio de la educación y el desarrollo integral.

Al aplicar la evaluación en el proceso de enseñanza aprendizaje se debe evitar que los estudiante sean pasivos, repetitivos e individualista; que las evaluaciones dejen de asumirse como autoritaria, que contengan un fuerte componente basado en el criterio del docente con respecto a los contenidos, evitar reactivos subjetivos, científicas, y temporales.

Al aplicar de forma incorrecta la evaluación, se puede detectar efectos negativos, tales como: rendimiento y deterioro de la calidad de educación, desprestigio institucional, no desarrollo de destrezas y habilidades en el estudiante.

Se debe concientizar a los docentes de inglés, sobre el modelo constructivista a fin de crear un espacio y el tiempo que permitan el diálogo y la reflexión de docentes, estudiantes y padres de familia.

De igual manera es necesario que los docentes motiven a los estudiantes, para generar una apertura en la renovación curricular, como fundamento para una práctica pedagógica investigativa. 
El apoyo e interés del docente es importante a fin de renovar y fortalecer su práctica pedagógica, adquiriendo material didáctico y fuentes bibliográficas que enriquezcan la fundamentación.

Fortalecer espacios de actualización y encuentros de docentes que permitan el intercambio y la reflexión, el docente en el proceso de enseñanza debe utilizar un conjunto de métodos, técnicas para llegar a un conocimientos científico, valorando y evaluado la capacidad del estudiante, desarrollando habilidades y destrezas; descartando la pasividad, el conformismo, el enciclopedismo, el autoritarismo y el individualismo en el estudiante.

La evaluación debe incidir en el proceso de aprendizaje entendiéndola como integral y dirigida hacia los tres tipos de contenidos: conceptuales, procedimentales y actitudinales. Sin duda se debe priorizar los criterios de evaluación de cada etapa, a fin de determinar la situación de estudiante en relación a una conducta perfectamente definida.

Desde el punto de vista educativo, la evaluación no solo se debe tener el carácter formativo, pues se debe entenderla a sí misma como un medio para facilitar la enseñanza de sus participantes, atendiendo en la elaboración de diseños

\section{Conclusion}

La evaluación del proceso de enseñanza aprendizaje del idioma inglés en los novenos años de Educación Básica de Unidad Educativa Julio María Matovelle mantiene características de1 modelo pedagógico tradicional; como evaluación de solo resultados, el alumno reproduce los conocimientos trasmitidos por el profesor, las pruebas se diseñan con el propósito de promover al alumno, sin tomar en cuenta el desarrollo de habilidades y destrezas.

No se evalúa las habilidades del pensamiento fundamental como capacidades de síntesis, nivel de razonamiento lógico, como habilidad para observar o para relacionar, para comprender la lectura de del inglés, Se trabaja únicamente en el nivel conceptual, dejando de lado el procedimental y actitudinal.

No hay relación entre metodología y evaluación; como los contenidos que pueden ser evaluados en forma grupal, se lo realiza de manera individual. Además, la evaluación del comportamiento del estudiante, lo cual implica cierto nivel de coerción, a fin de mantener la disciplina en el aula

Los maestros investigados, no llegan a ser mediadores o facilitadores del proceso de enseñanza aprendizaje, así lo refleja su casi nula participación en la solución de problemas de los alumnos y en la constante motivación a los mismos. 


\section{Recomendaciones}

En la evaluación del proceso de enseñanza aprendizaje del idioma Inglés se debe desterrar las características del modelo pedagógico tradicional; implementando un proceso científico intencional que se valga de ciertos instrumentos para estimar el valor de los resultados de un programa o actividad. En este proceso el maestro y su alumno deben determinar si han logrado los objetivos del proceso de enseñanza aprendizaje.

Establecer un sistema de comunicación que permita a los evaluados desarrollar las habilidades del pensamiento fundamentales como son capacidad de síntesis, nivel de razonamiento lógico, habilidad para observar o para relacionar, para comprender la lectura escritura del inglés. La evaluación debe realizarse en los tres niveles: conceptual, procedimental y actitudinal; creando prácticas evaluativas renovadoras como: organizadores gráficos, portafolios, exhibiciones y presentaciones creativas, autoevaluación y evaluación entre pares, diarios y bitácoras de aprendizaje, auto informes, cuestionarios, observación y sociometrías.

Se recomienda que la metodología y evaluación a aplicarse en cada contenido del idioma inglés tengan relación y permitan que el estudiante pueda construir su propio conocimiento. El comportamiento del alumno debe ser evaluado como medida de coerción; el maestro debe lograr empatía con sus alumnos, además debe motivarlos para que sus clases sean atractivas, lo cual implica, dominio de grupos, de contenidos, de estrategias metodológicas y de una profunda mística docente. Por lo tanto, se hace necesario la capacitación de los maestros tomando en cuenta estos lineamientos

\section{References:}

1. ALLEN, D., Ed. (2000). La evaluación de los aprendizajes de los estudiantes. Una herramienta para el desarrollo profesional de los docentes. Barcelona, Paidós.

2. BAIN, K. (2006), Lo que hacen los mejores profesores universitarios. Valencia: Publicacions Universitat de València.

3. BARBERÁ, E. (1999). Evaluación de la enseñanza, evaluación del aprendizaje. Barcelona, Edebé.

4. BOEKAERTS, M., Pintrich, P.; Zeidner, M. (Eds.) (2000). Handbook of self-regulation. Nueva York: Academic Press.

5. CRONBACH, L. J., \& Gleser, G. C. (1965). Psychological tests and personnel decisions. Oxford, England: U. Illinois Press

6. DOCHY, F., Segers, M. y Dierick, S. (2002). Nuevas Vías de Aprendizaje y Enseñanza y sus Consecuencias: Una Nueva Era de Evaluación. Boletín de la Red Estatal de Docencia Universitaria, 2, 2, 13-29. 
7. ESPASA (2002). Siglo XXI, Varios Autores. Enciclopedia de la Pedagogía, Tomo I.

8. GIROUX, Henry (1992). Teoría y resistencia en educación. Una metodología para la oposición, México, Siglo XXI, /UNAM

9. HALL, K. \& Burke, W. (2003). Making formative assessment work Effective practice in the primary classroom. Maidenhead, UK: Open University Press.

10. KAFTAN, J.; Buck, G.; Haack, A. (2006). Using Formative Assessments to Individualize Instruction and Promote Learning. Middle School Journal, 37, 4, 44-49.

11. MCDONALD, R,; Boud, D.; Francis, J. y Gonczi, A. (2000). Nuevas perspectivas sobre la evaluación. Boletín Cinterfor, 149, 41-72.. 